politische Entscheidungen dann bedeutsam, so Gstöhls Argument, wenn die Bewahrung der nationalen Identität in einem Staat durch zu starke Integrationsbestrebungen - seien sie ökonomisch noch so reizvoll - in Gefahr zu geraten scheint. In mehr als 30 empirischen Fallstudien zu politischen Integrationsentscheidungen zeigt die Autorin, dass insbesondere die Schweiz und Norwegen mit erheblich stärkeren sozio-politischen Restriktionen und Identitätsproblemen zu kämpfen hatten als Schweden. Anhand der Untersuchung von Referendumskampagnen, Wahlen und Umfragen verdeutlicht die Studie beispielsweise, dass die Integrationsentscheidungen in den 1990er Jahren in der Schweiz oft von der Sorge der schweizerischen Bevölkerung geprägt waren, mit einem Bekenntnis zur Europäischen Union gleichsam eigenständige demokratische Traditionen und das Prinzip der außenpolitischen Neutralität aufgeben zu müssen (198 f.). In Norwegen entbrannte der Streit um eine weit gehende Integration vor allem an der Ausgestaltung von Fischereirechten (196). Dabei gelang es den Integrationsgegnern, die Debatte um diesen traditionellen norwegischen Wirtschaftszweig zu instrumentalisieren, eine Abstimmungskoalition zwischen der Land- und Stadtbevölkerung Norwegens zu schmieden und damit die Integrationsbefürworter aus den Wirtschaftskreisen $\mathrm{zu}$ isolieren. Schwedens Integrationspolitik war dagegen von geringeren politischen Hindernissen geprägt, unter anderem deshalb, weil die schwedische Gesellschaft im Vergleich zur norwegischen keine Konfliktlinien auf Grund der historischen Erfahrung von Fremdherrschaft entwickelte.

Sieglinde Gstöhl hat mit ihrem Buch eine empirisch äußerst reichhaltige Studie zu einem bisher wenig beachteten Forschungsfeld vorgelegt. Ihr Buch besticht zudem durch methodische Genauigkeit und Transparenz. Auf der theoretischen Ebene ließe sich allerdings fragen, warum für die theoretische Thematisierung der Nachfragedimension der europäischen Integration auf eine Auseinandersetzung mit dem Neo-Funktionalismus vollständig verzichtet wurde. Denn mit diesem stünde eine theoretische Konzeption bereit, welche die Nachfrageseite der Integration - zumindest auf einer Makroebene - a priori berücksichtigt.
Jan Hasse, Erwin Müller, Patricia Schneider (Hg.): Menschrechte. Bilanz und Perspektiven. Baden-Baden: Nomos Verlagsgesellschaft 2002, 628 S., € 45,-.

Erwin Müller, Patricia Schneider, Kristina Thony (Hg.): Menschenrechtsschutz. Politische Maßnahmen, zivilgesellschaftliche Strategien, humanitäre Interventionen. BadenBaden: Nomos Verlagsgesellschaft 2002, 387 S., $€ 42,-$.

\section{Antje Lüdemann}

Die Menschenrechte bleiben trotz (oder wegen?) ihres Anspruchs auf universelle Gültigkeit ein umstrittenes Thema in der heutigen Welt-Gesellschaft. Nicht zuletzt durch Militäreinsätze, wie im Kosovo, in Afghanistan oder zukünftig eventuell im Irak, die fallweise als humanitäre Intervention zum Schutze der Menschenrechte legitimiert werden, gewinnt diese Problematik immer wieder Aktualität. So bleibt sie auch in der wissenschaftlichen Diskussion ein Thema, das viele bewegt. Die Thematik reizt, im Hinblick auf aktuelle Geschehnisse, alte Fragestellungen wieder neu aufzuwerfen.

Die Bände II „Menschenrechte“ und III „Menschenrechtsschutz" der Reihe „Frieden durch Recht" (Band I befasst sich mit dem humanitären Völkerrecht) widmen sich der Thematik der Menschenrechte in einer Zweiteilung: Im Band II „Menschenrechte“ werden die Menschenrechte als Errungenschaft der $\mathrm{Zi}$ vilisation bilanziert und diskutiert, während in Band III „Menschenrechtsschutz" deren Durchsetzung und die dafür gebrauchten Mittel im Vordergrund stehen. Die Autoren der Beiträge sind sowohl Rechts- und Sozialwissenschaftler als auch Vertreter von internationalen und Menschenrechtsorganisationen und tragen durch ihren jeweiligen Horizont zu einer sehr unterschiedlich pointierten Diskussion verschiedener Aspekte der Menschenrechtsproblematik bei.

Die Menschenrechte als höchstes vorstaatliches Recht und fundamentaler Grundsatz des modernen Völkerrechts sind der Ausgangspunkt der Beiträge in Band II „Menschenrechte". Das Ziel des Buches besteht darin, die Problematik dieser Rechte und ihrer Anerkennung in einer von Gewalt geprägten Welt aufzuzeigen. Dementsprechend gehen vier thematisch 